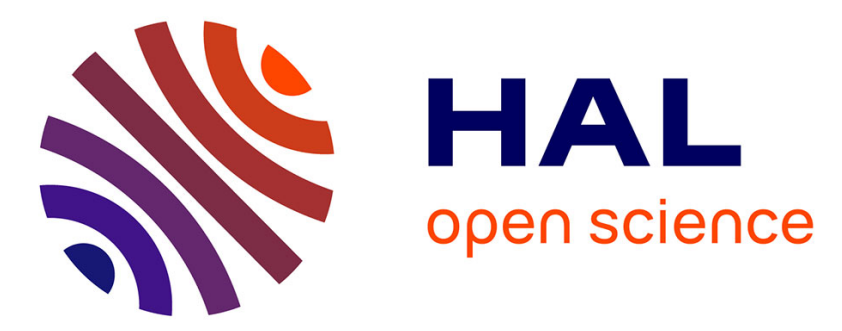

\title{
Single-molecule tautomerization tracking through space- and time-resolved fluorescence spectroscopy
}

Benjamin Doppagne, Tomáš Neuman, Ruben Soria-Martinez, Luis E Parra

López, Hervé Bulou, Michelangelo Romeo, Stéphane Berciaud, Fabrice

Scheurer, Javier Aizpurua, Guillaume Schull

\section{To cite this version:}

Benjamin Doppagne, Tomáš Neuman, Ruben Soria-Martinez, Luis E Parra López, Hervé Bulou, et al.. Single-molecule tautomerization tracking through space- and time-resolved fluorescence spectroscopy. Nature Nanotechnology, 2020, 15 (3), pp.207-211. 10.1038/s41565-019-0620-x · hal-02945947

\section{HAL Id: hal-02945947 \\ https://hal.science/hal-02945947}

Submitted on 22 Sep 2020

HAL is a multi-disciplinary open access archive for the deposit and dissemination of scientific research documents, whether they are published or not. The documents may come from teaching and research institutions in France or abroad, or from public or private research centers.
L'archive ouverte pluridisciplinaire HAL, est destinée au dépôt et à la diffusion de documents scientifiques de niveau recherche, publiés ou non, émanant des établissements d'enseignement et de recherche français ou étrangers, des laboratoires publics ou privés. 


\section{Single-molecule tautomerization tracking through space- and time-resolved fluorescence spectroscopy}

Benjamin Doppagne ${ }^{1}$, Tomáš Neuman ${ }^{2}$, Ruben Soria-Martinez ${ }^{1}$, Luis E.

Parra López ${ }^{1}$, Hervé Bulou ${ }^{1}$, Michelangelo Romeo ${ }^{1}$, Stéphane Berciaud ${ }^{1}$

Fabrice Scheurer ${ }^{1}$, Javier Aizpurua ${ }^{2}$, Guillaume Schull ${ }^{1 *}$

${ }^{1}$ Université de Strasbourg, CNRS, IPCMS, UMR 7504, F-67000 Strasbourg, France,

${ }^{2}$ Center for Materials Physics (CSIC-UPV/EHU) and DIPC, Paseo

Manuel de Lardizabal 5, Donostia - San Sebastián 20018, Spain.

(Dated: December 16, 2019)

PACS numbers: 
Tautomerization, the interconversion between two constitutional molecular isomers, is ubiquitous in nature ${ }^{1}$, plays a major role in chemistry ${ }^{2}$, and is perceived as an ideal switch function for emerging molecular-scale devices ${ }^{3}$. Within free-base porphyrin ${ }^{4}$, porphycene ${ }^{5}$ or phthalocyanine ${ }^{6}$, this process involves the concerted or sequential hopping of the two inner hydrogen atoms between equivalent nitrogen sites of the molecular cavity. Electronic and vibronic changes ${ }^{6}$ resulting from this NH-tautomerization, as well as details of the switching mechanism, were extensively studied with optical spectroscopies, even with singlemolecule sensitivity ${ }^{7}$. The influence of atomic-scale variations of the molecular environment and sub-molecular spatial resolution of the tautomerization could only be investigated using scanning probe microscopies ${ }^{3,8-11}$, at the expense of detailed information provided by optical spectroscopies. Here, we combine these two approaches, scanning tunneling microscopy (STM) and fluorescence spectroscopy ${ }^{12-15}$, to study the tautomerization within individual free-base phthalocyanine $\left(\mathrm{H}_{2} \mathrm{Pc}\right)$ molecules deposited on a NaCl-covered $\mathrm{Ag}(111)$ single crystal surface. STM-induced fluorescence (STM-F) spectra exhibit duplicate features that we assign to the emission of the two molecular tautomers. We support this interpretation by comparing hyper-resolved fluorescence maps ${ }^{15-18}$ (HRFMs) of the different spectral contributions with simulations accounting for the interaction between molecular excitons and picocavity plasmons ${ }^{19}$. We identify the orientation of the molecular optical dipoles, determine the vibronic fingerprint of the tautomers, and probe the influence of minute changes in their atomic-scale environment. Time-correlated fluorescence measurements allow us to monitor the tautomerization events and to associate the proton dynamics to a switching two-level system. Finally, optical spectra acquired with the tip located at a nanometer-scale distance from the molecule show that the tautomerization reaction occurs even when the tunneling current does not pass through the molecule. Together with other observations, this remote excitation indicates that the excited state of the molecule is involved in the tautomerization reaction path.

A sketch of the experiment is given in Fig. 1(a) where the silver-covered tungsten tip of a cryogenic STM is used to excite the fluorescence of a single $\mathrm{H}_{2} \mathrm{Pc}$ molecule (Fig. 1(b)) deposited on $\operatorname{Ag}(111)$ covered by three layers of $\mathrm{NaCl}$. A differential conductance $(\mathrm{d} I / \mathrm{d} V)$ 


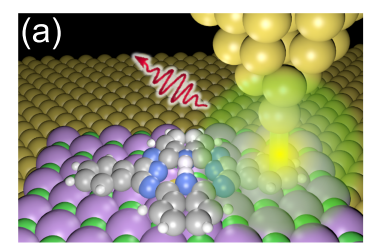

(b)

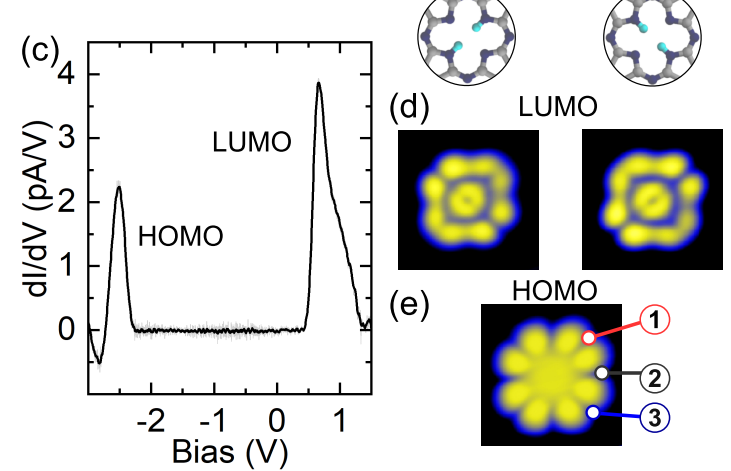

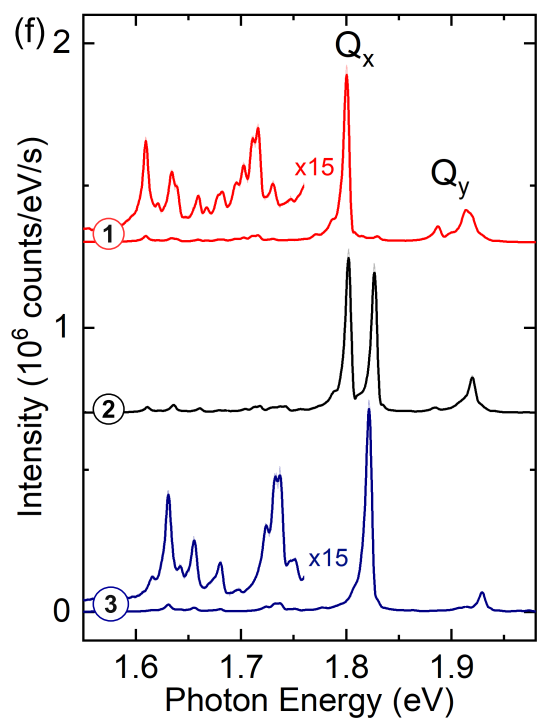

FIG. 1: STM-induced fluorescence spectroscopy of individual $\mathbf{H}_{2} \mathbf{P c}$ molecules. (a) Sketch of the STM-induced emission experiment. (b) Ball-and-stick model of the free-base phthalocyanine $\left(\mathrm{H}_{2} \mathrm{Pc}\right)$ and zoom on the central part of the molecule highlighting the structure of the two tautomers. (c) $\mathrm{d} I / \mathrm{d} V$ spectrum acquired on a single $\mathrm{H}_{2} \mathrm{Pc}$ adsorbed on 3 layers of $\mathrm{NaCl}$ on $\operatorname{Ag}(111)$. STM images $\left(3 \times 3 \mathrm{~nm}^{2}, I=10 \mathrm{pA}\right)$ acquired at $(\mathrm{d}) V=0.55 \mathrm{~V}$ and $(\mathrm{e}) V=-2.5$ V. Two patterns tilted by $90^{\circ}$ from each other may be observed in the image of a same molecule recorded at $V=0.55 \mathrm{~V}$ (d) corresponding to the two tautomers. (f) STM-F spectra acquired ( $V=$ $-2.5 \mathrm{~V}, I=100 \mathrm{pA}$, acquisition time $t=120 \mathrm{~s}$ ) for the STM tip located at the positions identified in $(\mathrm{e})$.

spectrum (Fig. 1(c)) recorded on a $\mathrm{H}_{2} \mathrm{Pc}$ molecule reveals the energy positions of the highest occupied and lowest unoccupied molecular orbitals $\left(\mathrm{E}_{H O M O} \approx-2.5 \mathrm{eV}\right.$ and $\left.\mathrm{E}_{L U M O} \approx 0.7 \mathrm{eV}\right)$. This assignment is confirmed by STM images recorded at the energies of the two spectral contributions (Fig. 1(d) and (e)) that can be directly compared to Density Functional Theory (DFT) calculations of the spatial projections of the HOMO and LUMO orbitals of $\mathrm{H}_{2} \mathrm{Pc}$ (section S1 in supplementary information). The LUMO may exhibit two similar two-fold symmetry patterns (Fig. 1(d)), rotated by $90^{\circ}$, that can be assigned ${ }^{20}$ to the two different tautomers (Fig. 1(b)). For a given molecule, a change of the pattern is often observed after a scan of the molecule at a negative voltage. The HOMO, however, always reveals the same four-fold symmetry image. STM-F spectra recorded at negative voltage $(V=-2.5 \mathrm{~V})$ for three different positions (noted 1, 2 and 3 in Fig. 1(e)) of the STM tip with respect to the 
$\mathrm{H}_{2} \mathrm{Pc}$ molecule are displayed in Fig. 1(f). Spectrum (1) shows an intense emission peak at 1.80 $\mathrm{eV}$, a weaker peak at $1.92 \mathrm{eV}$ and a series of very weak vibronic features on the low energy side of the main peaks. Based on a comparison with the literature ${ }^{6,14}$, the peaks at $1.80 \mathrm{eV}$ and $1.92 \mathrm{eV}$ can be assigned to the two first optical transitions of the $\mathrm{H}_{2} \mathrm{Pc}$ molecule named $Q_{x}$ and $Q_{y}$, respectively. $Q_{x}$ and $Q_{y}$ correspond to transition dipole moments oriented along and perpendicularly to the two inner hydrogen atoms of $\mathrm{H}_{2} \mathrm{Pc}$, respectively. The spectrum acquired on the same molecule in position (3) (Fig. 1(f)) shows a very similar spectral structure but with a $20 \mathrm{meV}$ shift of the $Q_{x}$ peak to higher energies. Eventually, spectrum (2) acquired in-between two benzopyrrole units of the $\mathrm{H}_{2} \mathrm{Pc}$ molecule, shows that this shift results from a duplication of the main spectral feature. Fluorescence spectra exhibiting a reduced energy separation between the duplicated peaks or no separation at all were also recorded for other $\mathrm{H}_{2} \mathrm{Pc}$ molecules. The presence or absence of duplicate features in the spectra results from minute-changes in the environment of the $\mathrm{H}_{2} \mathrm{Pc}$ molecules caused by their adsorption on different sites of the $\mathrm{NaCl} / \mathrm{Ag}(111)$ Moiré pattern ${ }^{21}$ (details in section S2 in supplementary information).

To unveil a potential connection between the duplicate spectra and the presence of two different tautomers, we acquired HRFMs of the molecule. We recorded a grid of STM-F spectra following the approach developed in [15-18]. A HRFM shows the spatial variation of the photon intensity associated to a given spectral feature with sub-molecular spatial resolution. The HRFMs are acquired with a constant tip-sample separation (i.e. open feedback loop) to prevent distance-related artifacts and are normalized by the tunneling current to have constant excitation intensities (see Methods). In Fig. 2(b, c) we display the HRFMs of four light emission contributions, noted $Q_{x 1}, Q_{x 2}, Q_{y 1}$ and $Q_{y 2}$ in the spectrum in Fig. 2(a). All the HRFMs show bright features on two opposite sides of the molecule that are separated by a darker region. Superimposed on these bright and dark regions, one also identifies features that are reminiscent of the HOMO pattern (section S1 in supplementary information). In Fig. 2(b, c) we also display theoretical simulations of the HRFMs characteristic of the $Q_{x}$ and $Q_{y}$ transition dipole moments of the $\mathrm{H}_{2} \mathrm{Pc}$ molecule. These simulations estimate the current-normalized fluorescence intensity for a given tip position by accounting for the product of the probability to drive the molecule in an excited state and the probability for this excitation to decay radiatively (details in section S3 in supplementary information). Our simulations confirm that the excitation efficiency is larger when electron tunneling takes 


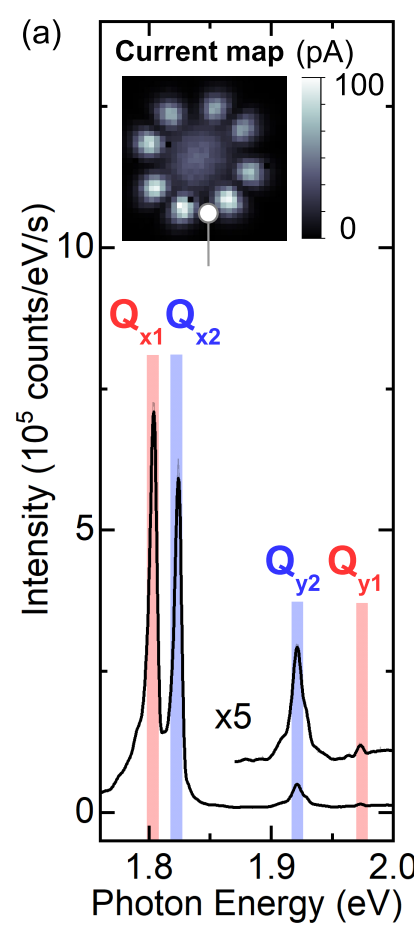

(b)
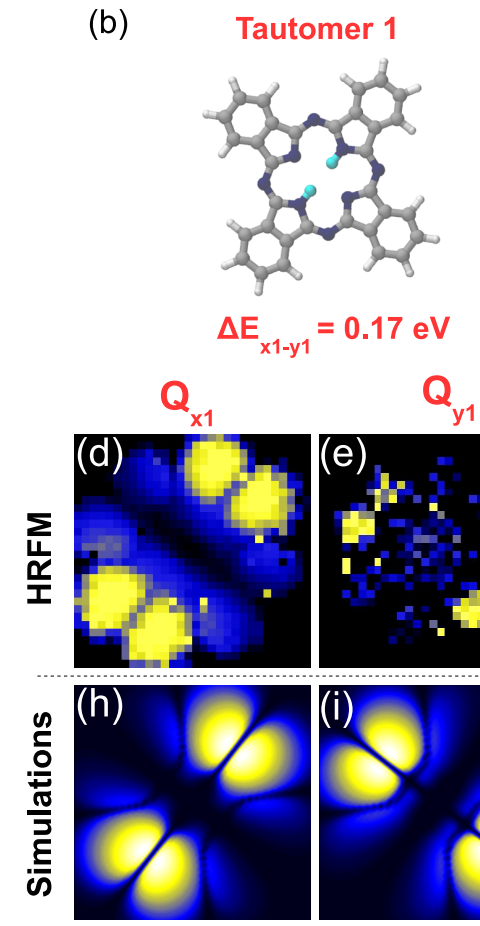

(c)
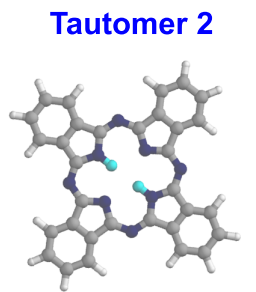

$$
\Delta E_{x 2-y 2}=0.10 \mathrm{eV}
$$
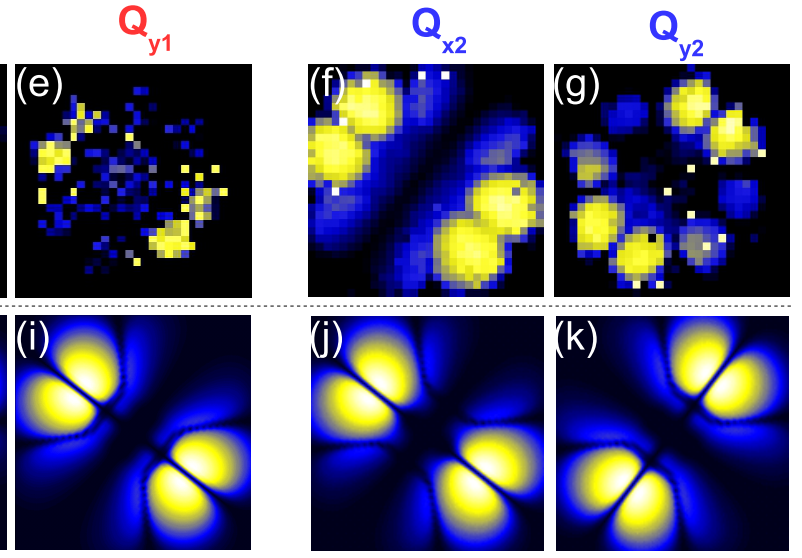

FIG. 2: Highly-resolved fluorescence mapping of a single $\mathbf{H}_{2} \mathbf{P c}$ molecule. (a) STM-F spectrum $(V=-2.5 \mathrm{~V}, I=100 \mathrm{pA}$, acquisition time $t=180 \mathrm{~s})$ acquired at the position marked by a circle in the current image $\left(2.5 \times 2.5 \mathrm{~nm}^{2}, V=-2.5 \mathrm{~V}\right)$ in inset. In (b) and (c) we display the chemical structure of tautomers 1 and 2, from $(\mathrm{d})$ to $(\mathrm{g})$ the associated HRFMs $(2.5 \times 2.5$ $\mathrm{nm}^{2}$, acquired simultaneously with the current map in (a), acquisition time $t=50 \mathrm{~s} /$ pixel, $30 \times$ 30 pixels) and from $(\mathrm{h})$ to $(\mathrm{k})$ their related simulated maps for the main contributions $\left(Q_{x 1}, Q_{x 2}\right.$, $Q_{y 1}$ and $Q_{y 2}$ ) identified in (a). Photon energy integration interval in (d) $1.798<\mathrm{h} \nu<1.811 \mathrm{eV}$, (e) $1.972<\mathrm{h} \nu<1.981 \mathrm{eV}$, (f) $1.824<\mathrm{h} \nu<1.832 \mathrm{eV}$ and (g) $1.923<\mathrm{h} \nu<1.941 \mathrm{eV}$.

place through the HOMO. This is in agreement with the increased fluorescence intensity observed when the bias is driven to resonance with the HOMO of the molecule ${ }^{14}$. This is also compatible with both the recent many-body state description of the excitation mechanism proposed by Miwa et al. that involves a charging step of the molecule by electron removal from the $\mathrm{HOMO}^{22}$, and an enhanced energy transfer between a tunneling electron and a molecular exciton ${ }^{13,18,23}$ that would occur for voltages driven at resonance with the HOMO. The radiative decay probability, on the other hand, is estimated by following a method described in details in [? ], by calculating the coupling strength between the picocavity plasmons $^{19}$, i.e., electromagnetic modes confined to an atomic-scale volume at the apex of 
the plasmonic tip, and the $Q_{x}$ and $Q_{y}$ transition dipole moments of $\mathrm{H}_{2} \mathrm{Pc}$. As observed previously ${ }^{15,16,24}$ this coupling results in an increased (attenuated) emission intensity for the tip localized at the extremities (center) of the molecular dipoles. In our case, the extreme spatial localisation of the field is responsible for the close to atomic-scale spatial resolution in the fluorescence maps. The excellent agreement between experimental and theoretical maps suggests that there are two series of $Q_{x}$ and $Q_{y}$ contributions corresponding to the two tautomers of the $\mathrm{H}_{2} \mathrm{Pc}$ molecules.

To emit at different energies, the two tautomers, that are otherwise equivalent, must experience slightly different environments ${ }^{6,25}$. This is confirmed by the substantially different $Q_{x}-Q_{y}$ gaps of the two tautomers. While this gap is pretty close to what is observed in cryogenic matrices $^{6}$ for tautomer 2 , it is much larger for tautomer 1 . This environment difference also affects the vibronic spectra (spectra (1) (blue) and (2) (red) in Fig. 1(f)). A detailed comparison of these vibronic fingerprints with a Raman spectrum obtained on a macroscopic crystal of $\mathrm{H}_{2} \mathrm{Pc}$ (section $\mathrm{S} 4$ in supplementary information) confirms that tautomer 2 is weakly affected by its adsorption site while the geometry of tautomer 1 is likely strained. This interpretation is supported by time-dependent DFT calculations of the $\mathrm{H}_{2} \mathrm{Pc}$ molecule that reveal similar variations of the $Q_{x}-Q_{y}$ gap for artificial compression (tension) of $5 \%(10 \%)$ of the molecule size along the $Q_{x}$ axis $\left(Q_{y}\right.$ axis) (section S5 in supplementary information). This combined spatial and spectral optical approach allows us to identify minute changes in the electronic structure and the geometry of two conformers that cannot be measured separately. The $\mathrm{NaCl} / \mathrm{Ag}(111)$ Moiré pattern (section S2 of the supplementary information) is likely responsible for this small variation of the tautomer environments.

Assigning the spectral contributions to two different tautomers also implies a fast switching of the molecule between these two conformations during the acquisition of the spectra and the HRFMs. To track the tautomerization dynamics directly through the STM-F spectra, we separated the collected light in two paths which were filtered at the energies of the $Q_{x 1}$ and $Q_{x 2}$ contributions, respectively, and eventually funneled to avalanche photodiodes (APDs) having a dead time of $\approx 100 \mathrm{~ns}$ (Fig. 3(a)). In Fig. 3(b) we display $200 \mathrm{~ms}$ of the time traces of the $Q_{x 1}$ and $Q_{x 2}$ emission lines (Fig. 3 (c)) recorded simultaneously with the tunneling current. These traces reveal alternating intense and weak emission periods for the two light contributions. Besides, the two traces seem to be in anti-phase, i.e., $Q_{x 1}$ is intense when the $Q_{x 2}$ is weak and vice versa. Eventually, we note that these variations 
(a)

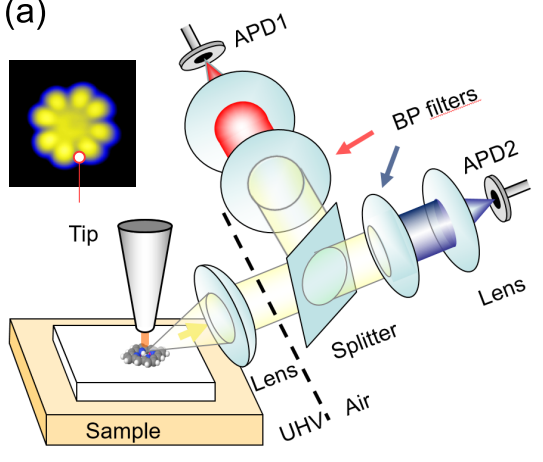

(b)

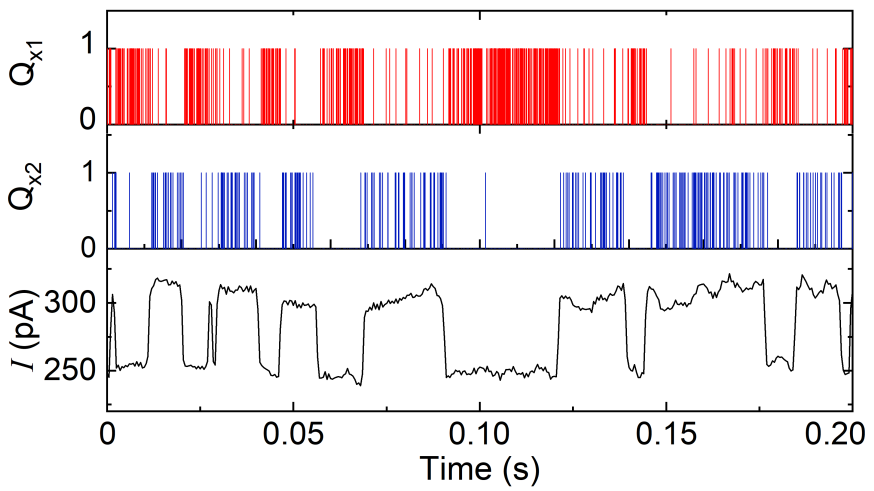

(c)

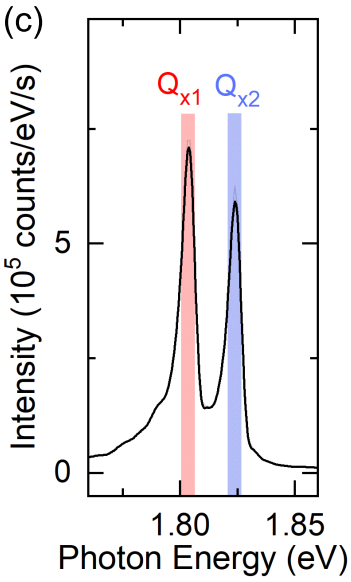

(d)

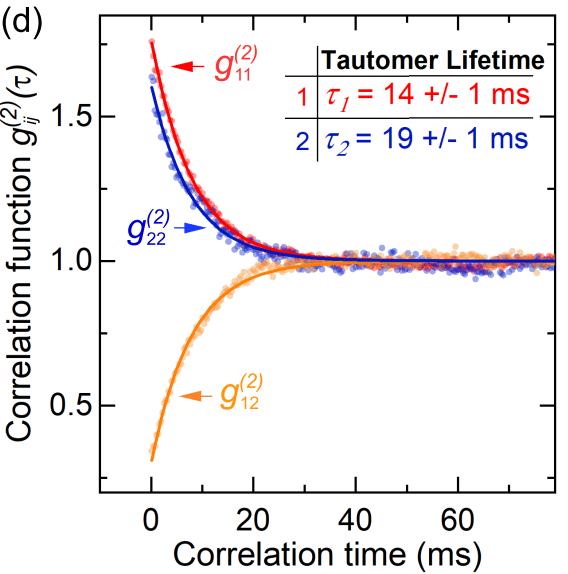

(e)

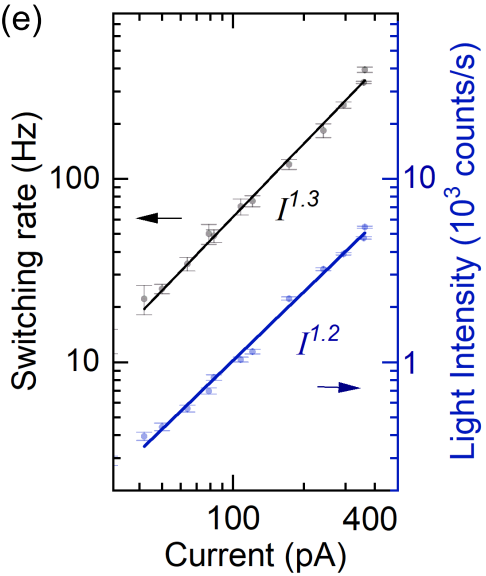

FIG. 3: Tautomerization dynamics reveald by spectrally-filtered time-correlated fluo-

rescence. (a) Sketch of the time-resolved setup. STM image of the molecule $(V=-2.5 \mathrm{~V}, I=$ $10 \mathrm{pA}, 3.5 \times 3.5 \mathrm{~nm}^{2}$ ) (b) Time traces of the signal recorded simultaneously on APD1 and APD2, corresponding to the $Q_{x 1}$ and $Q_{x 2}$ contributions, respectively, and of the tunnel current $(V=-2.5$ $\mathrm{V}$ ). The tip position is indicated by a dot in the STM image in (a). For this position of the tip, STM-F spectra (c) display intense $Q_{x 1}$ and $Q_{x 2}$ contributions. (d) Second order autocorrelation $\left(g_{i i}^{(2)}\right)$ and cross-correlation function $\left(g_{i j}^{(2)}\right)$ of the signal measured by the APDs. (e) Plot of the switching rate $k$ as deduced from fits of the $g^{(2)}$ functions and of the light intensity (for $V=-2.5$ V) as a function of the tunneling current.

match fluctuations observed in the tunneling current that could then be assigned to changes of the local conductance for the two tautomers.

To confirm and quantify these observations, we follow a fluorescence correlation spectroscopy approach ${ }^{26}$ that has been used in combination with STM recently to characterize molecular fluctuations ${ }^{27,28}$ and single-photon sources ${ }^{29,30}$. We display in Fig. 3(d) the second-order auto-correlation functions $g_{i i}^{(2)}(\tau)=\left\langle I_{Q_{x i}}(\tau) I_{Q_{x i}}(t+\tau)\right\rangle /\left\langle I_{Q_{x i}}(t)\right\rangle^{2}$ and the 

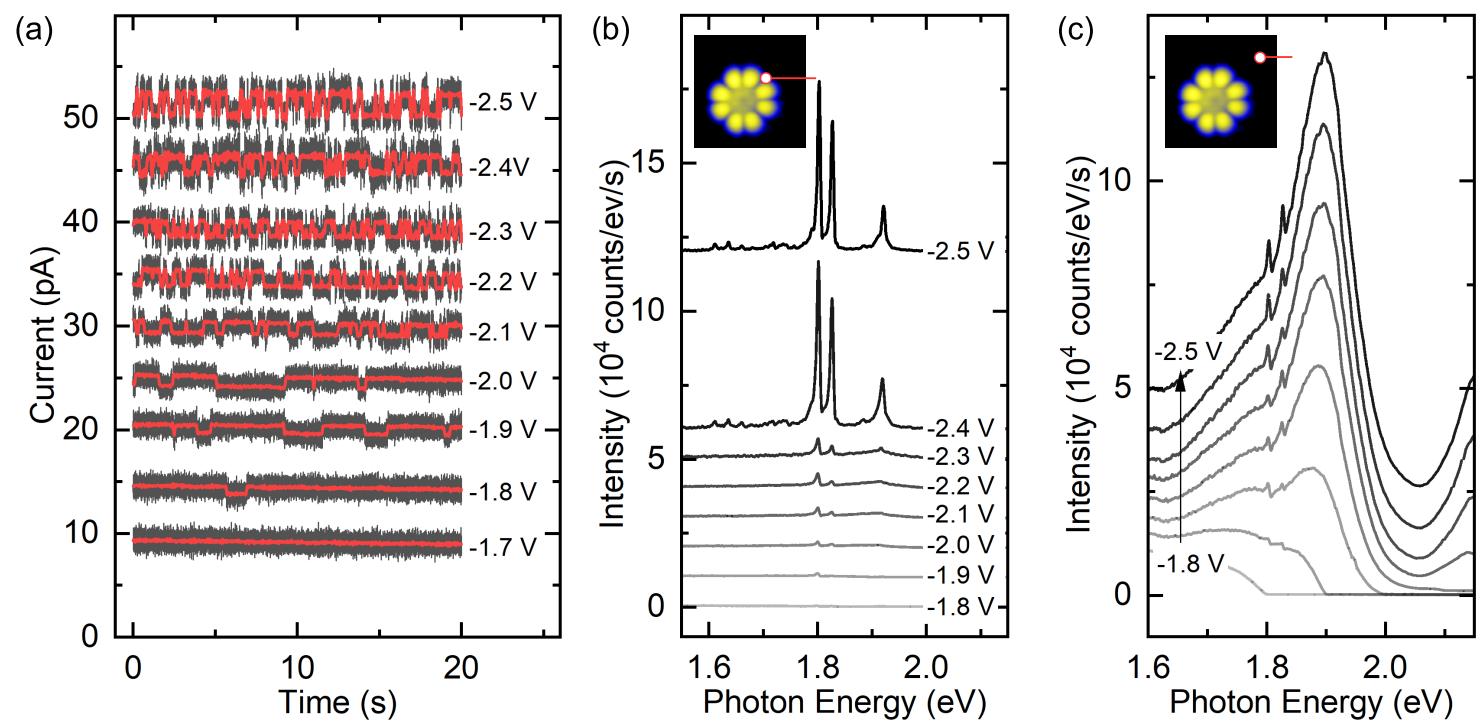

FIG. 4: Excited-state mediated tautomerization reaction. (a) Current-time traces (initial setpoint $I=10 \mathrm{pA})$ and (b, c) STM-F spectra $(I=20 \mathrm{pA}(\mathrm{b})$ and $I=600 \mathrm{pA}(\mathrm{c}))$ as a function of $V$. Current-traces and spectra are vertically offset for clarity. The position of the tip in (a) and (b) (respectively (c)) is marked by a dot in the STM image $\left(V=-2.5 \mathrm{~V}, I=10 \mathrm{pA}, 4 \times 4 \mathrm{~nm}^{2}\right)$ in the inset of (b) (respectively (c)).

second-order cross-correlation function $g_{12}^{(2)}(\tau)=\left\langle I_{Q_{x 1}}(\tau) I_{Q_{x 2}}(t+\tau)\right\rangle /\left\langle I_{Q_{x 1}}(t)\right\rangle\left\langle I_{Q_{x 2}}(t)\right\rangle$ of the fluorescence intensity $\left(I_{Q_{x i}}\right)$ of the $Q_{x 1}$ and $Q_{x 2}$ contributions. The $g_{11}^{(2)}(\tau)$ and $g_{22}^{(2)}(\tau)$ functions allow us to estimate the probability of observing pairs of photons ${ }^{26}$ separated by a time $\tau$ on APD 1 and 2, respectively, while the $g_{12}^{(2)}(\tau)$ function characterizes the probability to detect a $Q_{x 2}$ photon at a time $\tau$ after the detection of a $Q_{x 1}$ photon. For short time separations, the auto-correlation functions reveal a bunched emission $\left(g_{i i}^{(2)}(\tau)>1\right)$, whereas an anti-bunching is evidenced in the cross-correlation function $\left(g_{12}^{(2)}(\tau)<1\right)$. For larger values of $\tau$, an uncorrelated emission $\left(g^{(2)}(\tau)=1\right)$ characterizes both functions. Several conclusions may be drawn from these results. The presence of an anti-bunching in the $g_{12}^{(2)}(\tau)$ function confirms that the $Q_{x 1}$ and $Q_{x 2}$ contributions belong to two configurations that do not appear at the same time, thus validating the hypothesis of a switching between the two tautomeric forms. The bunching observed in the $g_{i i}^{(2)}(\tau)$ supports this interpretation and directly reflects the presence of time laps during which the molecule remains in a given tautomer. Moreover, we deduce from tip-position dependent correlation measurements that the time spent by the molecule in the tautomer 2 configuration is consistently longer than in 
the tautomer 1 configuration (section S6 in supplementary information), which agrees well with the more stable character of the tautomer 2 configuration.

The $g_{i i}^{(2)}$ functions can be very well fitted with a single exponential decay: $g_{i i}^{(2)}(\tau)=$ $1+A \exp (-k \tau)$ where $k$ is the tautomer switching frequency ${ }^{26}$. We show in Fig. 3(e) that the dependency of $k$ with tunneling current is slightly super-linear. This is somehow surprising in the frame of the electron-activated process generally considered in this case ${ }^{3}$. Besides, we see (Fig. 3(e)) that the tautomerization rate follows almost the same current dependency as the fluorescence intensity. Interestingly, a similar correlation exists between the voltage dependencies of these two processes. Indeed, a reduction of the switching rate is observed in current traces recorded at lower absolute voltages (Fig. 4(a)), and is beyond detection for voltages $\geq-1.7 \mathrm{~V}$. It is at this same voltage threshold that we start to observe (Fig. 4(b)) the fluorescence of the molecule. This correlation may indicate an activation mechanism where the tautomerization reaction occurs through an excitation/deexcitation cycle of the molecule. To possibly identify such an effect, we recorded fluorescence spectra for the same voltage sequence (Fig. $4(\mathrm{c})$ ) but with the tip located $\approx 1.2 \mathrm{~nm}$ laterally away from the molecule (see STM image in inset). In this configuration, it has been shown that the molecule can still be driven to its excited state through energy transfer between the tunneling electron and the molecular exciton ${ }^{20,24,31}$. In contrast, processes directly activated by tunneling electrons traversing the molecule, such as charging ${ }^{32}$, cannot occur. The data of Fig. 4(c) show that both $Q_{x 1}$ and $Q_{x 2}$ contributions are observed in all spectra acquired with $V<-1.8 \mathrm{eV}$, implying that the tautomerization occurs even in the absence of direct tunneling through the molecule. Together with the current (Fig. 3(e)) and voltage (Fig. 4(a)) dependencies, this indicates an excited-state proton transfer, similarly to what was deduced from laser-excitation experiments ${ }^{33}$. This conclusion is further supported by similar data acquired on single free-base naphthalocyanine molecules that have a lower optical gap and consequently revealed a tautomerization onset at a lower voltage (section S7 of the supplementary information). As fluorescence information was not available, this mechanism was not considered in previous tautomerization experiments induced by $\mathrm{STM}^{3,8,10,34,35}$ (see section $\mathrm{S} 7$ for a detailed discussion of the tautomerization mechanism). This mechanism may also be involved in other STM-activated phenomena such as diffusion, rotation, dissociation or switching of single molecules on surface.

We used hyper-resolved fluorescence microscopy in combination with time-correlated mea- 
surements and spectral selection to identify and characterize a tautomerization reaction within a prototypical single-molecule switch. We obtained detailed information on the interaction of the molecule and the substrate in the two tautomeric conformations, on the dynamics of the hydrogen atoms within the molecule, and on the activation mechanism of the reaction in the STM junction. We believe that the unraveled reaction path, which involves the excited state of the molecule, may also play an important role in several other STM-activated experiments where it has not been considered. Moreover, by developing a simulation method that accounts for both the excitation and deexcitation probabilities in a STM-F experiment, we make an important step towards a rationalization of hyper-resolved fluorescence maps, i.e., the fluorescence microscopy approach that provides the highest spatial resolution to date. Eventually, and in contrast with other experiments where molecular switches are shown to modulate only the current traversing a nanoscale circuit, we demonstrate that a molecular optoelectronic element can also be used to activate an optical signal.

\section{METHODS}

The experiments were performed with a low temperature (4.5 K) Omicron STM operating under ultrahigh vacuum. The microscope was complemented with an optical setup capable of detecting the light emitted at the tip-sample junction. The emitted photons are collected with a lens located in the vacuum chamber at $4.5 \mathrm{~K}$ and then transferred out of the chamber. To characterize the spectral dependency of the emitted light and for the hyper-resolved fluorescence maps, the light is focused on an optical fiber that is connected to a spectrograph coupled to a CCD camera. Two optical gratings of 300 and 1200 grooves were used resulting in spectral resolutions of $\approx 0.7 \mathrm{~nm}$ (spectra of Fig. 1, Fig. 2, Fig. 3, Fig. 4, Fig. S6, Fig. S11, Fig. S12, Fig. S13) and $\approx 0.2 \mathrm{~nm}$ (spectra of Fig. S2, and Fig. S3) respectively. The details of the optical setup are described in the Supplemental Material of [13]. For the time-resolved measurements (see also Fig. 3(a)) the photon stream is divided in two lines by a 50/50 splitter, filtered using bandpass filters (bandwidth of $10 \mathrm{~nm}$ centered at $676 \mathrm{~nm}$ and $690 \mathrm{~nm}$ ) and eventually focused on two avalanche photodiodes (Excelitas SPCM-AGRHFC). The STM tips were prepared from electrochemically etched tungsten wire. In situ, they were prepared by $\mathrm{Ar}^{+}$sputtering and annealing. The tips were eventually indented in the sample to cover them with a thin silver layer in order to optimise their plasmonic 
response. The $\operatorname{Ag}(111)$ substrates were cleaned by several sputtering and annealing cycles. $\mathrm{NaCl}$ was evaporated on the $\mathrm{Ag}(111)$ sample which was held around $300 \mathrm{~K}$. The molecules were deposited on the sample at low temperature $(\approx 10 \mathrm{~K})$ from a powder placed in a quartz crucible. The Raman spectrum (Fig. S6 (b)) were acquired at resonance on a $\mathrm{H}_{2} \mathrm{Pc}$ crystal at room temperature and in air (excitation wavelength $632.8 \mathrm{~nm}$ ). Hyper-resolved fluorescence maps (HRFMs) were generated by scanning the targeted molecule with the STM tip while recording a STM-F spectrum for each tip position (i.e., pixel of the map). The tip-molecule separation was kept constant during the acquisition (open feedback loop) to prevent any distance-related artifacts. To compensate for drifts of the tip during the long acquisition of the HRFMs, the (x, y, z) position of the tip was corrected using an "atom-tracking" procedure between the acquisition of each pixel, similarly to the procedure developed in $[?]$. The next step consists in choosing the photon energy windows corresponding to the spectral feature of interest, namely $Q_{x 1}, Q_{x 2}, Q_{y 1}$ and $Q_{y 2}$, and to generate the corresponding photon intensity maps. By this method, all the HRFMs were recorded simultaneously in a single experimental run and readily compared. Eventually, the photon intensity maps were normalized by the STM current map recorded simultaneously. Indeed, as the tunneling current acts in this experiment as an excitation source and since the current varies as a function of tip position, normalizing for each pixel the photon intensity by the corresponding tunneling current allows us to generate maps with constant excitation source intensity.

\section{DATA AVAILABILITY}

The data that support the plots within this paper and other findings of this study are available from the corresponding author upon reasonable request.

\section{ACKNOWLEDGEMENTS}

The authors thank Virginie Speisser for technical support and Alex Boeglin for fruitful discussions. This project has received funding from the European Research Council (ERC) under the European Union's Horizon 2020 research and innovation program (grant agreement No 771850). The Agence National de la Recherche (project SMALL'LED No. ANR-14-CE26-0016-01), the Labex NIE (Contract No. ANR-11-LABX-0058_NIE), and the 
International Center for Frontier Research in Chemistry (FRC) are acknowledged for financial support. R. S. M. and H. B. acknowledge GENCI-CINES (Project No. A0060907459) and the Pôle HPC and Equipex Equip@Meso at the University of Strasbourg. T.N. and J. A. acknowledge the project FIS2016-80174-P from the Spanish Ministry of Science, and project ELKARTEK KK-2018/00001 from the Basque Government, as well as grant IT1164-19 from the Basque Governement for consolidated groups at the university..

\section{AUTHOR CONTRIBUTIONS}

B.D. and G.S. conceived, designed and performed the experiments. B.D., M.R., F.S. and G.S. analysed the experimental data. T.N. and J.A. conceived and performed the simulations of the HRFM maps. R.S.M. and H.B. performed the DFT calculations of the $\mathrm{H}_{2} \mathrm{Pc}$ under strain. L.E.P.L and S.B. performed and analyzed the Raman spectra. All the authors discussed the results and contribute to the redaction of the paper.

\section{ADDITIONAL INFORMATION}

Supplementary information is available in the online version of the paper. Reprints and permission information is available online at www.nature.com/reprints. Correspondence and requests for materials should be addressed to [G.S.]

1 Abou-Zied, O. K., Jimenez, R. \& Romesberg, F. E. Tautomerization dynamics of a model base pair in dna. J. Am. Chem. Soc. 123, 4613-4614 (2001).

2 Antonov, L. Tautomerism - methods and theories. (Wiley: Weinheim, Germany, 2014, 2014).

3 Liljeroth, P., Repp, J. \& Meyer, G. Current-induced hydrogen tautomerization and conductance switching of naphthalocyanine molecules. Science 317, 1203 (2007).

4 Storm, C. B. \& Teklu, Y. Nitrogen-hydrogen tautomerism in porphyrines and chlorines. J. Am. Chem. Soc. 94, 1745-1747 (1972).

5 Waluk, J. Spectroscopy and tautomerization studies of porphycenes. Chem. Rev. 117, 2447$2480(2017)$. 
${ }^{6}$ Murray, C. et al. Visible luminescence spectroscopy of free-base and zinc phthalocyanines isolated in cryogenic matrices. Phys. Chem. Chem. Phys. 13, 17543-17554 (2011).

7 Chizhik, A. M. et al. Optical imaging of excited-state tautomerization in single moleculesw. Phys. Chem. Chem. Phys. 13, 1722-1733 (2011).

8 Kumagai, T. et al. Thermally and vibrationally induced tautomerization of single porphycene molecules on a cu(110) surface. Phys. Rev. Lett. 111, 246101 (2013).

9 Kumagai, T. et al. Controlling intramolecular hydrogen transfer in a porphycene molecule with single atoms or molecules located nearby. Nature Chemistry 6, 41 (2013).

10 Ladenthin, J. N. et al. Force-induced tautomerization in a single molecule. Nature Chem. 8, 935 (2016).

11 Kügel, J., Klein, L., Leisegang, M. \& Bode, M. Analyzing and tuning the energetic landscape of h2pc tautomerization. J. Phys. Chem. C 121, 28204-28210 (2017).

12 Qiu, X. H., Nazin, G. V. \& Ho, W. Vibrationally resolved fluorescence excited with submolecular precision. Science 299, 542-546 (2003).

13 Chong, M. C. et al. Narrow-line single-molecule transducer between electronic circuits and surface plasmons. Phys. Rev. Lett. 116, 036802 (2016).

14 Imada, H. et al. Real-space investigation of energy transfer in heterogeneous molecular dimers. Nature 538, 364-367 (2016).

15 Zhang, Y. et al. Visualizing coherent intermolecular dipole-dipole coupling in real space. Nature 531, 623 (2016).

16 Chen, C., Chu, P., Bobisch, C. A., Mills, D. L. \& Ho, W. Viewing the interior of a single molecule: Vibronically resolved photon imaging at submolecular resolution. Phys. Rev. Lett. 105, $217402(2010)$.

17 Lee, J., Perdue, S. M., Rodriguez Perez, A. \& Apkarian, V. A. Vibronic motion with joint angstrom-femtosecond resolution observed through fano progressions recorded within one molecule. ACS Nano 8, 54-63 (2014).

18 Doppagne, B. et al. Vibronic spectroscopy with submolecular resolution from stm-induced electroluminescence. Phys. Rev. Lett. 118, 127401 (2017).

19 Urbieta, M. et al. Atomic-scale lightning rod effect in plasmonic picocavities: A classical view to a quantum effect. ACS Nano 12, 585-595 (2018). PMID: 29298379.

20 Imada, H. et al. Single-molecule investigation of energy dynamics in a coupled plasmon-exciton 
system. Phys. Rev. Lett. 119, 013901 (2017).

21 Pivetta, M., Patthey, F., Stengel, M., Baldereschi, A. \& Schneider, W.-D. Local work function moiré pattern on ultrathin ionic films: Nacl on ag(100). Phys. Rev. B 72, 115404 (2005).

22 Miwa, K. et al. Many-body state description of single-molecule electroluminescence driven by a scanning tunneling microscope. Nano Letters 19, 2803-2811 (2019).

23 Chong, M. C. et al. Ordinary and hot electroluminescence from single-molecule devices: Controlling the emission color by chemical engineering. Nano Lett. 16, 6480-6484 (2016).

24 Kröger, J., Doppagne, B., Scheurer, F. \& Schull, G. Fano description of single-hydrocarbon fluorescence excited by a scanning tunneling microscope. Nano Lett. 18, 3407-3413 (2018).

25 Macfarlane, R. \& Völker, S. A comparison of phototautomerism in different sites of free-base porphin (h2p) in n-alkane crystals. Chem. Phys. Lett. 69, $151-155$ (1980).

${ }^{26}$ Lippitz, M., Kulzer, F. \& Orrit, M. Statistical evaluation of single nano-object fluorescence. ChemPhysChem 6, 770 (2005).

27 Perronet, K., Schull, G., Raimond, P. \& Charra, F. Single-molecule fluctuations in a tunnel junction: A study by scanning-tunnelling-microscopy-induced luminescence. EPL (Europhysics Letters) 74, 313 (2006).

28 Merino, P. et al. A single hydrogen molecule as an intensity chopper in an electrically driven plasmonic nanocavity. Nano Lett. 19, 235-241 (2019).

29 Merino, P., Große, C., Rosławska, A., Kuhnke, K. \& Kern, K. Exciton dynamics of c60-based single-photon emitters explored by hanbury brown-Â"twiss scanning tunnelling microscopy. Nat. Commun. 6, 8461 (2015).

30 Zhang, L. et al. Electrically driven single-photon emission from an isolated single molecule. Nat. Commun. 8, 580 (2017).

31 Zhang, Y. et al. Sub-nanometre control of the coherent interaction between a single molecule and a plasmonic nanocavity. Nat. Commun. 8, 15225 (2017).

32 Doppagne, B. et al. Electrofluorochromism at the single-molecule level. Science 361, 251 (2018).

33 Völker, S. \& van der Waals, J. Laser-induced photochemical isomerization of free base porphyrin in an n-octane crystal at 4.2 k. Mol. Phys. 32, 1703-1718 (1976).

34 Kügel, J. et al. Remote single-molecule switching: Identification and nanoengineering of hot electron-induced tautomerization. Nano Letters 17, 5106-5112 (2017). PMID: 28732159. 
35 Böckmann, H. et al. Near-field enhanced photochemistry of single molecules in a scanning tunneling microscope junction. Nano Letters 18, 152-157 (2018). 